\section{La restriction calorique chez les primates}

Julia Marchal, Martine Perret, Fabienne Aujard

> La restriction calorique est la seule intervention non génétique connue à ce jour pour ralentir l'apparition de pathologies liées à l'âge et augmenter la durée de vie moyenne et maximale chez plusieurs espèces. Son intérêt ne cesse de croître en particulier pour l'identification des mécanismes qui interviennent dans l'augmentation de la longévité. Contrairement aux études chez les modèles invertébrés et les rongeurs, qui ont permis de fournir certaines indications sur les mécanismes de la restriction calorique, l'efficacité de cette dernière en tant que protocole antivieillissement chez les primates n'a pas encore été totalement démontrée. Dans cette synthèse, nous présentons les avantages de l'utilisation des primates non humains comme modèles pertinents pour l'étude du vieillissement humain en général, et plus particulièrement dans le contexte d'interventions thérapeutiques applicables à l'homme, telles que la restriction calorique. En nous appuyant notamment sur des résultats longitudinaux chez le microcèbe (Microcebus murinus), nous soulignons l'importance des études sur les primates dans le contexte des recherches sur le vieillissement. Par ailleurs, nous présentons le potentiel de développement de molécules qui miment les effets bénéfiques constatés de la restriction calorique, permettant ainsi d'en reproduire les effets positifs, déjà constatés chez certaines espèces, sans imposer un régime hypocalorique. <

Malgré de nombreux témoignages et anecdotes concernant les bienfaits d'une vie « sobre » où l'apport énergétique est réduit, l'utilisation de ce paradigme nutritionnel dans un contexte de recherche fondamentale ou appliquée est plus tardive. Ce n'est qu'en 1935 qu'une

Vignette : microcèbe (Photo (c) Éric Gueton).

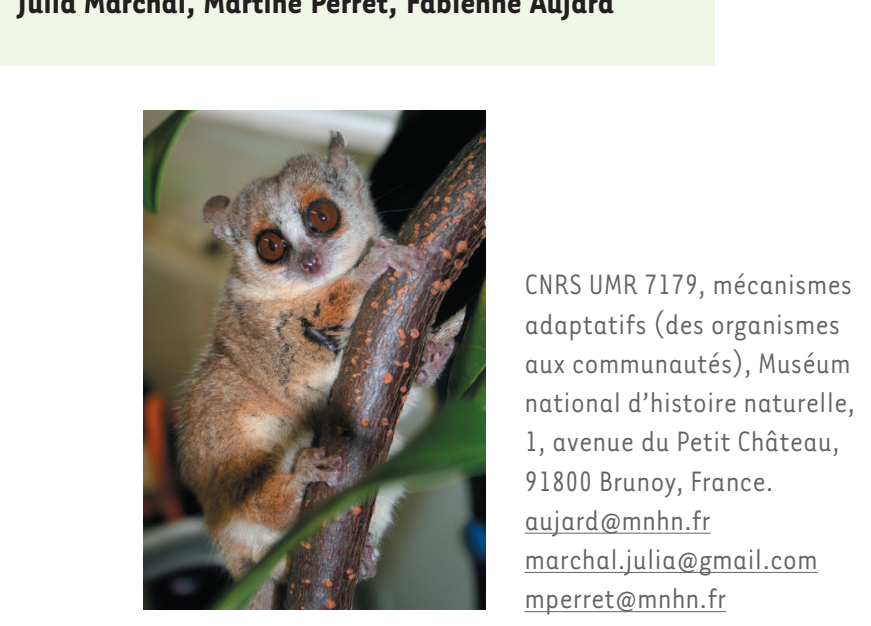

première étude menée chez le rat par McCay et al. [1] met en évidence qu'une restriction calorique de 40 \% au moment du sevrage entraîne une augmentation de l'espérance de vie chez les animaux restreints. Les conclusions de cette étude ont agi comme un véritable promoteur puisque de nombreuses études ont alors vu le jour chez d'autres espèces animales. Une restriction calorique correspond à une diminution de la prise alimentaire sans malnutrition, c'est-à-dire sans induction d'excès ou de carence pour un ou plusieurs nutriments composant le régime alimentaire de base [2]. Des organismes unicellulaires aux mammifères, la restriction calorique a des effets similaires sur l'augmentation de la longévité associée à un retard d'apparition de pathologies liées à l'âge et à un maintien des fonctions biologiques de l'organisme. De plus, de nombreuses études rapportent les effets bénéfiques d'un tel traitement sur un grand nombre de pathologies chroniques liées à l'âge, telles que le diabète de type 2, l'obésité, les maladies cardiovasculaires, les déficiences du système immunitaire, l'artériosclérose, l'hypertension artérielle et les cancers [3]. Les données actuelles invitent à émettre I'hypothèse selon laquelle des espèces différentes répondent de manière similaire à une diminution des disponibilités alimentaires en allongeant leur durée de vie. Ceci suggère que les réponses de l'organisme lors d'une restriction calorique dépendent d'un ou de mécanisme(s) également impliqué(s) dans la «lutte » contre le vieillissement qui est(sont) partagé(s) par des espèces provenant de différents ordres. Plusieurs voies métaboliques ont été proposées pour l'explication de tels effets bénéfiques [4]. La restriction calorique pourrait donc améliorer la qualité de vie et augmenter la longévité de l'espèce humaine [5]. Bien que quelques données rapportent des effets bénéfiques chez l'homme, les études sur des sujets humains sont plutôt rares au vu de la longévité de notre espèce. Par ailleurs, les modèles fréquemment utilisés, comme les invertébrés et les rongeurs, peuvent présenter des limites liées, d'une part, à leur éloignement phylogénétique par rapport à l'homme et, 


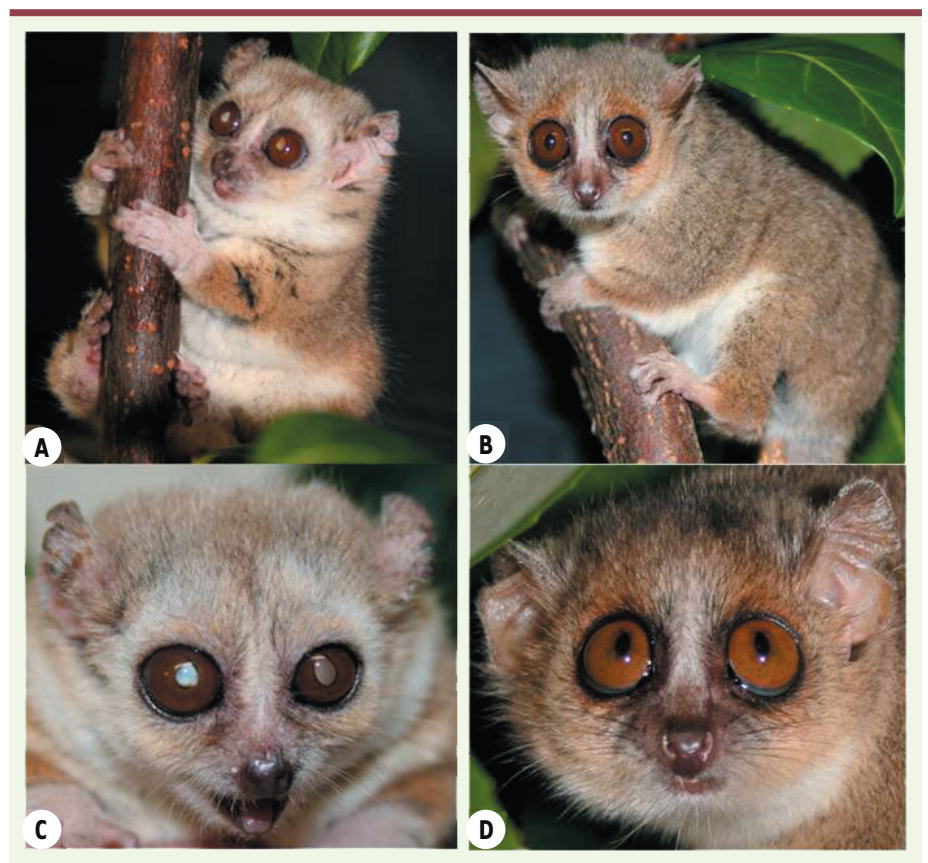

Figure 1. Photographies de microcèbes du projet Restrikal. (A) et (C) Aspect morphologique à l'âge de sept ans d'un animal du groupe contrôle, nourri ad libitum. (B) et (D) Aspect morphologique à l'âge de sept ans d'un animal du groupe soumis à une restriction calorique de $30 \%$ par rapport au groupe contrôle (@ Photo Éric Gueton).

d'autre part, à l'absence de l'expression naturelle de certaines pathologies liées à l'âge et pouvant être affectées par une restriction calorique. Il est donc primordial de valider des modèles expérimentaux adaptés aux études de la restriction calorique, mais également à l'application de cette intervention à des fins thérapeutiques chez l'homme. Plus récemment, des études sur des primates non humains ont été débutées $\rightarrow$ [50]. L'utilisation de ces modèles phylogénétiquement proches de l'homme semble être un bon compromis qui permet d'appréhender les bases

$\rightarrow$ Voir la Brève de Julia Marchal et al., page 1074 de ce numéro sous-jacentes au vieillissement et les voies impliquées lors d'une restriction calorique, outils nécessaires au développement de mimétiques de cette restriction calorique. En effet, pour des raisons sociales, économiques et médicales, une restriction calorique semble difficilement applicable chez les humains, en particulier à long terme. Ainsi, un panel de molécules candidates, répondant aux critères de mimétiques et pouvant stimuler les mêmes voies d'actions cellulaires que la restriction calorique, font actuellement l'objet d'études visant à retrouver les effets bénéfiques provoquées par une restriction calorique chronique et modérée, sans imposer un régime hypocalorique.

\section{Les effets de la restriction calorique chez les primates non humains}

Les effets de la restriction calorique chez les macaques

Alors que les modèles invertébrés et vertébrés ont permis la mise en évidence de certains mécanismes d'action de la restriction calorique, particulièrement à l'échelle moléculaire et cellulaire, des études se sont développées sur des primates non humains, principalement le macaque rhésus Macaca mulatta, le singe écureuil Saimiri sciureus ou le microcèbe Microcebus murinus [48]. Les primates représentent un lien important entre la recherche fondamentale et l'application clinique de protocoles thérapeutiques visant à améliorer la santé de l'homme. Chez ces espèces, la méthode la plus répandue pour instaurer une restriction calorique nécessite d'abord de mesurer la prise alimentaire individuelle des animaux lorsqu'ils sont nourris ad libitum, c'est-à-dire lorsque la ressource alimentaire est disponible à volonté jusqu'à satiété. L'apport calorique est ensuite individuellement abaissé d'un pourcentage précis variant de 30 à 60 \% [2]. Il y a environ 25 ans, deux études ont débuté à l'Institut national du vieillissement (Baltimore, États-Unis) et à l'université du Wisconsin (Madison, États-Unis) [6] chez des macaques rhésus (Maccaca mulatta) auxquels était appliquée une restriction calorique de $30 \%$ à partir de l'âge adulte (qui est atteint à environ 10 ans chez cette espèce) [7]. À partir de l'âge de 20 ans environ, le pourcentage de survie des macaques contrôles décline plus rapidement que celui des macaques restreints. Après 15 ans de traitement, le taux de survie des macaques restreints est supérieur de $20 \%$ à celui des témoins [8]. L'apparition d'un diabète de type 2 et de maladies cardiovasculaires est deux fois moins fréquente chez les animaux en restriction calorique que chez les témoins, dont la moitié a développé des états prédiabétique ou diabétique [8]. Tout comme chez les rongeurs, les macaques soumis à une restriction calorique ont présenté des adaptations structurales, hormonales et métaboliques [9]. Notamment, ont été observées une diminution importante de la masse grasse des animaux, une sensibilité à l'insuline plus importante, ainsi qu'une diminution des dommages causés par les radicaux libres $^{1}$ [10] et des processus inflammatoires [11]. De plus, le déclin des fonctions immunitaires lié à l'âge, l'atrophie de la matière grise cérébrale, ainsi que l'apparition de sarcopénie ont également été atténués chez les animaux soumis à une restriction calorique [12]. Cette dernière induit chez les primates une diminution de la masse corporelle, une réduction de la masse grasse, mais également celle de la température corporelle par comparaison avec des animaux contrôles du même âge [13]. Cependant, l'impact de la restriction calorique sur les fonctions reproductrices des primates est moins clair. Chez le

\footnotetext{
${ }^{1}$ Ces dommages étaient mesurés au niveau des muscles squelettiques par microsco-
} pie électronique et marquage par anticorps des protéines altérées. 


\begin{tabular}{|lllll}
\hline \multicolumn{1}{|c}{$\begin{array}{c}\text { Paramètres } \\
\text { biologiques }\end{array}$} & $\begin{array}{c}\text { Microcèbe } \\
\text { (Microcebus } \\
\text { murinus) }\end{array}$ & \multicolumn{2}{c}{ Homme } \\
\hline Masse corporelle & $\searrow$ & {$[18]$} & $\searrow$ & {$[23]$} \\
\hline Activité locomotrice & $\nearrow$ & {$[19,20]$} & $\searrow$ & {$[25]$} \\
\hline Température corporelle & $\searrow$ & {$[19]$} & $\searrow$ & {$[27]$} \\
\hline Sensibilité à l'insuline & $\nearrow$ & {$[21]$} & $\nearrow$ & {$[23]$} \\
\hline $\begin{array}{l}\text { Capacités mnésiques } \\
\text { (mémoire de travail) }\end{array}$ & $\nearrow$ & {$[20]$} & ND & {$[30]$} \\
\hline
\end{tabular}

Tableau I. Effets d'une restriction calorique chez le microcèbe et chez l'homme. Comparaison de l'évolution de paramètres chez le microcèbe, un primate non humain et chez l'homme. Les références pour chaque paramètre sont entre crochets. ND : non déterminé.

macaque rhésus comme chez le rat, la restriction calorique retarde la puberté, et il semblerait qu'elle freine la croissance osseuse des animaux restreints [14]. Enfin, l'impact d'une restriction calorique sur le raccourcissement des télomères a été recherché chez les macaques. Le rôle du raccourcissement des télomères dans le vieillissement cellulaire est bien connu et, chez l'homme, des données épidémiologiques ont démontré que la longueur des télomères dans les leucocytes était extrêmement variable à la naissance mais ensuite inversement corrélée à l'âge. Chez les macaques, aucun effet de la restriction calorique sur la longueur des télomères n'a pu être encore mis en évidence [15]. Cependant, chez les primates communément utilisés (macaque, singeécureuil), les animaux âgés sont rares et leur longue durée de vie rend très difficile toute étude longitudinale, c'est-à-dire l'observation des mêmes individus à différents âges.

La restriction calorique ralentit le processus de vieillissement chez le microcèbe

Ces dernières années, un intérêt croissant s'est porté sur un petit primate, le microcèbe (Microcebus murinus), comme nouveau modèle primate du vieillissement [16]. La connaissance de ses caractéristiques de vie, le succès de son élevage en captivité, sa durée de vie relativement courte ( 8 à 10 ans) et la caractérisation de l'évolution de nombreux paramètres biologiques en fonction de l'âge (comme le métabolisme basal ou la masse corporelle [17]) en font un modèle très intéressant pour l'étude des altérations physiologiques, comportementales et cérébrales liées à l'âge chez les mammifères dont l'homme. Les microcèbes inclus dans l'étude RESTRIKAL, débutée en 2007, ont été soumis à deux régimes alimentaires différents à l'âge de trois ans : un régime contrôle et une restriction calorique modérée de $30 \%$. L'ensemble de leurs paramètres physiologiques et comportementaux ont été suivis en longitudinal jusqu'à leur mort naturelle. En comparaison d'animaux témoins nourris normalement, les différents marqueurs du vieillissement ont été modifiés par la restriction calorique. Après trois ans d'exposition à une restriction calorique (30\% par rapport aux animaux contrôles), les rythmes saisonniers, métaboliques et endocriniens caractéristiques de ce primate ont été conservés, confirmant ainsi l'adaptation à long terme des animaux à un tel traitement. La masse corporelle des animaux en restriction calorique est plus basse avec un écart important, particulièrement en milieu de la saison d'été [18]. D'un point de vue morphologique, après trois ans de traitement les animaux restreints paraissent plus jeunes que les animaux contrôles dont le poil au même âge est grisonnant, le museau et les oreilles raccourcies (Figure 1). Des perturbations des rythmes d'activité locomotrice, caractérisées en particulier par une fragmentation et une augmentation de l'activité pendant la période de sommeil diurne, sont classiquement observées chez les microcèbes âgés [17]. Après un an de traitement, la restriction calorique a significativement augmenté l'activité locomotrice nocturne spontanée chez les animaux restreints [18]. De même, une restriction calorique de 40 ou $80 \%$ pendant un mois a entraîné une diminution de la température corporelle chez les animaux restreints [19]. L'évaluation des capacités cognitives montre l'impact bénéfique de la restriction calorique en ce qui concerne la mémoire de «travail » (évaluée grâce au test de l'alternance spontanée) connue pour être moins sensible au vieillissement que la mémoire spatiale ou les fonctions exécutives. Cette capacité mnésique est altérée chez les animaux vieillissants, tandis que les animaux restreints présentent de bien meilleurs résultats après deux ans de traitement [20]. Enfin, une nette augmentation de la tolérance au glucose ainsi qu'une augmentation de la sensibilité à l'insuline chez les animaux restreints sont observées, résultats qui concordent avec ceux qui ont déjà été observés chez les rongeurs et les autres primates non humains [21]. L'ensemble des résultats acquis chez cette espèce, et qui présentent de fortes similitudes avec les données établies chez l'homme (Tableau 1), confortent l'hypothèse d'un ralentissement des processus du vieillissement et d'un retard d'apparition des altérations liées à l'âge chez les primates soumis à une restriction calorique modérée, à long terme.

\section{Et chez l'homme?}

Nous connaissons l'exemple des habitants de l'île d'0kinawa au Japon qui ont la plus longue espérance de vie ( 86 ans pour les femmes et 78 ans pour les hommes), ainsi que le plus grand nombre de centenaires : 34 centenaires pour 100000 habitants contre 11 pour 100000 habitants en France [22]. La longévité exceptionnelle des centenaires d'Okinawa est attribuable, en partie, à leur mode de vie dont une alimentation pauvre en gras 
et en calories. Chez l'homme, une restriction calorique modérée (25\%) de trois à six mois s'accompagne d'effets bénéfiques marqués contre l'obésité, la résistance à l'insuline, les mécanismes inflammatoires, le stress oxydatif et les dysfonctionnements cardiovasculaires [23], résultats en accord avec ceux obtenus chez des rongeurs subissant le même traitement $[25,49]$. Comme chez les rongeurs, des modifications hormonales sont observées chez l'homme soumis à une restriction calorique : augmentation de l'adiponectine, réduction de la triiodothyronine (hormone thyroïdienne impliquée dans la régulation du métabolisme), baisse des taux de testostérone et d'insuline. La restriction calorique permet aussi de diminuer un certain nombre de facteurs de risque impliqués dans le développement de maladies cardiovasculaires comme le taux de cholestérol, la quantité de protéine C-réactive (qui a un rôle important dans les réponses inflammatoires), la pression sanguine et l'épaisseur de l'intima-média de l'artère carotide [24]. Ce régime alimentaire diminue également la température corporelle des individus. Enfin, il induit des adaptations métaboliques et comportementales avec une baisse du niveau d'activité physique chez des volontaires soumis à une restriction calorique pendant 6 mois [25]. II est intéressant de noter qu'il existe des différences majeures dans les effets de la restriction calorique chez les primates et les rongeurs. Par exemple, une restriction calorique modérée induit une diminution de 30 à $40 \%$ du taux d'IGF-1 (insulin-like growth factor-1) plasmatique chez les rongeurs mais n'a aucun effet chez l'homme [26]. Dans l'étude Baltimore longitudinal study of aging (BLSA), une restriction calorique modérée et à long terme (six ans en moyenne) induit une diminution de la température corporelle moyenne de jour et de nuit chez l'homme [27], résultat également démontré chez des rats restreints [28] ou chez le macaque rhésus soumis à une restriction calorique de 30 \% [29]. Bien qu'un grand nombre d'arguments convergent pour souligner les effets bénéfiques d'une restriction calorique sur des paramètres physiologiques, les effets sur le comportement et les capacités cognitives chez l'homme sont encore très mitigés [30]. Par ailleurs des essais cliniques d'application d'une restriction calorique chez des patients en chimiothérapie ont montré des résultats encourageants [31]. En effet, la diminution d'un grand nombre d'effets secondaires liés à la thérapie, ainsi qu'une possible augmentation de son efficacité, ont été observées chez une dizaine de patients soumis à des jeûnes répétés pendant leur chimiothérapie.

\section{La restriction calorique et ses limites, vers quelles solutions?}

La restriction calorique s'accompagne d'un grand nombre de modifications physiologiques et comportementales, à l'échelle de la cellule et à l'échelle de l'organisme entier. Même si ses effets bénéfiques sont nombreux, elle peut également altérer certaines fonctions physiologiques, probablement via une diminution des ressources énergétiques. Par exemple, la cicatrisation de plaies cutanées est réduite chez des rats soumis à une restriction calorique de $40 \%$, cicatrisation qui peut être considérablement améliorée si les rats restreints sont nourris ad libitum 48 h avant de subir la lésion cutanée [32]. Des effets négatifs de la restriction calorique chez les rongeurs ont été démontrés : ils concernent la résistance au stress ou aux infections provoquées par des bactéries ou des vers parasites, même s'il est vrai que la restriction calorique permet de ralentir le déclin lié à l'âge de certaines fonctions immunitaires [33]. Chez les primates, l'augmentation de la sensibilité aux infections par des pathogènes ou le ralentissement du processus de cicatrisation n'ont pas encore été étudiés. Chez les macaques, la restriction calorique à long terme affecte négativement la minéralisation osseuse [14] et l'axe neuroendocrine de la reproduction [13]. Chez l'homme aussi, des effets délétères ont été rapportés, comme une diminution de la densité osseuse [34]. Finalement, malgré les avantages avérés de la restriction calorique, toutes les connaissances sur l'innocuité d'un tel régime, nécessaires pour le développement et l'application d'une restriction calorique à long terme chez l'homme, ne sont pas réunies.

\section{Les mimétiques en plein essor !}

Une approche alternative à la restriction calorique est donc aujourd'hui nécessaire. Le terme et le concept de mimétiques de la restriction calorique ont vu le jour à la fin des années 1990. Depuis l'émergence de ce concept, les recherches de mimétiques de la restriction calorique ne cessent d'augmenter. Une définition a d'ailleurs été proposée : «composé qui mime les effets métaboliques, hormonaux et physiologiques de la restriction calorique, active des voies d'actions similaires, améliore la résistance au stress oxydant, augmente la longévité, retarde l'apparition des pathologies liées à l'âge et maintient les fonctions de l'organisme dans un état jeune, sans réduire l'apport calorique » [35]. Plusieurs composés candidats répondent à ces critères et, actuellement, trois d'entre eux attirent beaucoup l'attention des scientifiques: les polyphénols, les inhibiteurs glycolytiques et certains composés cellulaires régulant l'expression génique.

Au cours des 15 dernières années, l'intérêt pour les bienfaits des composés polyphénoliques a beaucoup augmenté depuis que certaines études épidémiologiques ont suggéré qu'il existait une relation entre la consommation d'aliments riches en polyphénols et la prévention de certaines maladies, telles que les cancers, les maladies cardiovasculaires ou certaines maladies neurodégénératives comme la maladie d'Alzheimer [36]. Le resvératrol, composé phénolique naturel trouvé notamment dans la peau du raisin, est un des candidats les plus prometteurs quant à sa capacité à mimer les effets bénéfiques de la restriction calorique. Cette molécule de la famille des stilbènes présente plusieurs 
propriétés bénéfiques telles qu'une action anti-inflammatoire [37] et anticancérigène [38]. Elle procure également des effets bénéfiques chez des rats soumis à un régime riche en lipides en diminuant la masse corporelle, la pression artérielle et les pathologies cardiovasculaires [39]. Le thé possède aussi une grande quantité de polyphénols qui auraient des effets protecteurs contre certains cancers [40] et contre le développement de maladies cardiovasculaires [41].

Parallèlement, un analogue du sucre est à l'étude, le 2-déoxyglucose, qui agit comme un inhibiteur glycolytique. Cette molécule présente des effets similaires à la restriction calorique concernant la régulation métabolique puisqu'elle réduit les taux d'insuline et la température corporelle des rats traités [35]. Cependant, des études à long terme ont montré une certaine toxicité du 2-déoxyglucose, qui provoque une augmentation des pathologies cardiaques et de la mortalité chez le rat [42]. Un autre candidat prometteur en tant que mimétique de la restriction calorique est représenté par les inhibiteurs de la cible de la rapamycine chez les mammifères, mTOR (mammalian target of rapamycin). mTOR est une sérine thréonine kinase impliquée dans la régulation de la prolifération cellulaire, ainsi que dans la survie cellulaire. Une perturbation dans la voie de signalisation de mTOR induit des pathologies ainsi que des cancers [43]. Par ailleurs, I'utilisation de la rapamycine peut inhiber mTOR et empêcher dans certains cas la prolifération des cellules tumorales [44].

Enfin, l'AMPK (adenosine monophosphate-activated protein kinase), une protéine kinase activée par l'AMP, est également au centre des recherches concernant les mimétiques des effets bénéfiques de la restriction calorique, car elle est connue pour être un élément majeur de régulation du métabolisme énergétique cellulaire. Une activation pharmacologique de l'AMPK améliore à la fois la sensibilité au glucose, le métabolisme des lipides et la pression artérielle chez des rongeurs insulinorésistants [45]. De plus, une stimulation de I'AMPK engendre une diminution de la synthèse de certaines protéines, impliquées dans la régulation de la longévité, notamment par une inhibition de la voie de signalisation mTOR [46]. En outre, certaines molécules antidiabétiques, comme la metformine, ont pour cible cette protéine AMPK ainsi que mTOR, ce qui augmente leur intérêt pour les études cliniques évaluant des thérapies contre le syndrome métabolique ou le diabète de type $2[47]$.

\section{Conclusion}

Alors que les effets bénéfiques de la restriction calorique ont été démontrés pour la première fois chez l'animal il y a presqu'un siècle, l'amélioration des techniques et les différents modèles animaux utilisés dans ce domaine ont considérablement alimenté les bases mécanistiques de la restriction calorique. Ainsi, l'utilisation de cette intervention nutritionnelle dans un but thérapeutique, dans le cadre de la prévention des pathologies chroniques liées à l'âge, a rapidement permis, au cours de ces 20 dernières années, de visualiser l'étendue des effets bénéfiques via un nombre relativement faible de voies d'actions cellulaires impliquées dans des processus clés, tels que la régulation du métabolisme énergétique et le stress oxydant cellulaire. Bien que les effets positifs de la restriction calorique ne soient pas unanimement démontrés, les études sur les primates non humains se développent, ainsi que les études cliniques et épidémiologiques conduites chez l'homme. Le développement de molécules mimétiques de la restriction calorique qui pourraient pallier certaines limites de l'utilisation de ce régime, est actuellement en plein essor. Certains candidats, comme le resvératrol, sont de plus en plus étudiés chez les modèles animaux de restriction calorique. Malgré des résultats parfois mitigés, notamment quant à l'allongement de la durée de vie, ces molécules ont probablement devant elles un avenir thérapeutique. L'ensemble de ces études contribue largement à une meilleure compréhension de la biologie du vieillissement. De plus, l'avancée rapide dans le domaine de la biogérontologie et de la nutrition ouvre des voies sérieuses et prometteuses quant au développement de stratégies thérapeutiques applicables à l'homme. $\diamond$

\section{SUMMARY}

Caloric restriction in primates: how efficient as an anti-aging approach?

Caloric restriction (CR) is the only non-genetic intervention known to date to slow the onset of age-related diseases and increase average and maximum lifespan in several species. Its interest is continually growing, particularly for the identification of mechanisms involved in increasing longevity. Unlike studies in invertebrate and rodent models have provided some indication about the mechanisms of the CR, the efficacy of $C R$ as an anti-aging protocol in primates has not yet been fully established. In this review we present the advantages of using non human primates as relevant models to the study of human aging in general and specifically in the context of therapeutic interventions applicable to humans, such as CR. Through the longitudinal findings in the Grey Mouse Lemur (Microcebus murinus), we stress the importance of primate studies in the context of research on aging and their potential to advance the development of molecules which can mimic the beneficial effects of $C R$, already observed in some species, without imposing a reduced calorie diet. $\diamond$

\section{LIENS D'INTÉRÊT}

Les auteurs déclarent n'avoir aucun lien d'intérêt concernant les données publiées dans cet article.

\section{RÉFÉRENCES}

1. McCay CM, Crowell MF, Maynard LA. The effect of retarded growth upon the length of life span and upon the ultimate body size. J Nutrition 1935; 10 : 63-79.

2. Cantó C, Auwerx J. Caloric restriction, SIRTl and longevity. Trends Endocrinol Metab $2009 ; 20: 325-31$. 


\section{RéFéRENCES}

3. Speakman JR, Mitchell SE. Caloric restriction. Mol Aspects Med 2011 ; 32 : 159-221.

4. Fadini GP, Ceolotto G, Pagnin $\varepsilon$, et al. At the crossroads of longevity and metabolism: the metabolic syndrome and lifespan determinant pathways. Aging Cell $2011 ; 10: 10-7$.

5. Holloszy J0, Fontana L. Caloric restriction in humans. Exp Gerontol 2007 ; 42 : 709-12.

6. Kemnitz JW. Calorie restriction and aging in nonhuman primates. ILARJ $2011 ; 52: 66-77$.

7. Roth GS, Ingram DK, Lane MA. Calorie restriction in primates: will it work and how will we know? J Am Geriatr Soc 1999 ; $47: 896-903$

8. Colman RJ, Anderson RM, Johnson SC, et al. Caloric restriction delays disease onset and mortality in rhesus monkeys. Science $2009 ; 325: 201-4$.

9. Kemnitz JW, Roecker $\varepsilon B$, Weindruch $R$, et al. Dietary restriction increases insulin sensitivity and lowers blood glucose in rhesus monkeys. Am J Physiol $1994 ; 266$ : ع540-7.

10. Zainal TA, Oberley TD, Allison DB, et al. Caloric restriction of rhesus monkeys lowers oxidative damage in skeletal muscle. FASEB J $2000 ; 14: 1825-36$.

11. Lane MA, Ball SS, Ingram DK, et al. Diet restriction in rhesus monkeys lowers fasting and glucosestimulated glucoregulatory end points. Am J Physiol $1995 ; 268$ : ع94l-8.

12. Colman RJ, Beasley TM, Allison DB, Weindruch R. Attenuation of sarcopenia by dietary restriction in rhesus monkeys. J Gerontol A Biol Sci Med Sci 2008; $63: 556-9$.

13. Mattison JA, Lane MA, Roth GS, Ingram DK. Calorie restriction in rhesus monkeys. Exp Gerontol $2003 ; 38: 35-46$.

14. Roth GS, Ingram DK, Black A, Lane MA. Effects of reduced energy intake on the biology of aging: the primate model. Eur J Clin Nutr $2000 ; 54$ : S15-20.

15. Smith DL Jr, Mattison JA, Desmond RA, et al. Telomere dynamics in rhesus monkeys: no apparent effect of caloric restriction.J Gerontol A Biol Sci Med Sci 2011 ; 66 : 1163-8.

16. Languille $S$, Blanc $S$, Blin 0 , et al.The grey mouse lemur: a non-human primate model for ageing studies. Ageing Res Rev $2012 ; 11: 150-62$

17. Perret M, Aujard F. Vieillissement et rythmes biologiques chez les primates. Med Sci (Paris) 2006 ; $22: 279-283$.

18. Dal-Pan A, Terrien J, Pifferi F, et al. Caloric restriction or resveratrol supplementation and ageing in a non-human primate: first-year outcome of the RESTRIKAL study in Microcebus murinus. Age $2011 ; 33: 15-31$

19. Giroud S, Blanc S, Aujard F, et al. Chronic food shortage and seasonal modulations of daily torpo and locomotor activity in the grey mouse lemur (Microcebus murinus). Am J Physiol Regul Integr Comp Physiol 2008 ; 294 : R1958-67.

20. Dal-Pan A, Pifferi F, Marchal J, et al. Cognitive performances are selectively enhanced during chronic caloric restriction or resveratrol supplementation in a primate. Plos One $2011 ; 6: 16581$.

21. Marchal J, Blanc S, Epelbaum J, et al. Effects of chronic calorie restriction or dietary resveratrol supplementation on insulin sensitivity markers in a primate, Microcebus murinus. PLoS One 2012 $7:$ e34289.

22. Suzuki M, Willcox DC, Rosenbaum MW, Willcox BJ. Oxidative stress and longevity in okinawa: an investigation of blood lipid peroxidation and tocopherol in okinawan centenarians. Curr Gerontol Geriatr Res $2010 ; 380460$

23. Fontana L, Meyer TE, Klein S, Holloszy J0. Long-term calorie restriction is highly effective in reducing the risk for atherosclerosis in humans. Proc Natl Acad Sci USA 2004 ; 101 : 6659-63.

24. Fontana L, Villareal DT, Weiss EP, et al. Calorie restriction or exercise: effects on coronary heart disease risk factors. A randomized, controlled trial. Am J Physiol Endocrinol Metab 2007 ; 293 : ع197-202.

25. Redman LM, Ravussin $\varepsilon$. Caloric restriction in humans: impact on physiological, psychological, and behavioral outcomes. Antioxid Redox Signal $2011 ; 14$ : 275-87.

26. Fontana L, Weiss EP, Villareal DT, et al. Long-term effects of calorie or protein restriction on serum IGF-1 and IGFBP-3 concentration in humans. Aging Cell $2008 ; 7: 681-7$.

27. Soare $A$, Cangemi $R, 0$ modei $D$, et al. Long-term calorie restriction, but not endurance exercise, lowers core body temperature in humans. Aging (Albany NY) $2011 ; 3: 374-9$.

28. Duffy PH, Feuers RJ, Leakey JA, et al. Effect of chronic caloric restriction on physiological variables related to energy metabolism in the male Fischer 344 rat. Mech Ageing Dev $1989 ; 48$ 117-33.

29. Lane MA, Baer DJ, Rumpler WV, et al. Calorie restriction lowers body temperature in rhesus monkeys, consistent with a postulated anti-aging mechanism in rodents. Proc Natl Acad Sci USA $1996 ; 93: 4159-64$.
30. Mattson MP. The impact of dietary energy intake on cognitive aging. Front Aging Neurosci $2010 ; 2: 5$.

31. Raffaghello L, Safdie F, Bianchi G, et al. Fasting and differential chemotherapy protection in patients. Cell Cycle $2010 ; 9$ : 4474-6.

32. Hunt ND, Li GD, Zhu M, et al. Effect of calorie restriction and refeeding on skin wound healing in the rat. Age (Dordr) 2011 (online).

33. Kristan DM. Chronic calorie restriction increases susceptibility of laboratory mice (Mus musculus) to a primary intestinal parasite infection. Aging Cell $2007 ; 6: 817-25$

34. Riedt CS, Buckley BT, Brolin RE, et al. Blood lead levels and bone turnove with weight reduction in women. J Expo Sci Environ Epidemiol 2009 ; 19 : 90-6.

35. Roth GS, Lane MA, Ingram DK. Caloric restriction mimetics: the next phase. Ann Ny Acad Sci $2005 ; 1057$ : 365-71.

36. Di Stefano A, Iannitelli A, Laserra S, Sozio P. Drug delivery strategies for Alzheimer's disease treatment. Expert Opin Drug Deliv $2011 ; 8$ : 581-603.

37. González R, Ballester I, López-Posadas R, et al. Effects of flavonoids and other polyphenols on inflammation. Crit Rev Food Sci Nutr 2011 ; 51 : 33162.

38. Huang $X$, Zhu HL. Resveratrol and its analogues: promising antitumor agents. Anticancer Agents Med Chem 2011 ; 11 : 479-90.

39. Baur JA, Sinclair DA. Therapeutic potential of resveratrol: the in vivo evidence. Nature Rev Drug Discov 2006 ; 5 : 493-506.

40. Yang CS, Wang ZY. Tea and cancer. J Natl Cancer Inst 1993 ; 85 : 1038-49.

41. Tijburg LB, Wiseman SA, Meijer GW, Weststrate JA. Effects of green tea, black tea and dietary lipophilic antioxidants on LDL oxidizability and atherosclerosis in hypercholesterolaemic rabbits. Atherosclerosis 1997 ; $135: 37-47$.

42. Minor RK, Smith DL Jr, Sossong AM, et al. Chronic ingestion of 2-deoxy$\mathrm{D}$-glucose induces cardiac vacuolization and increases mortality in rats. Toxicol Appl Pharmacol $2010 ; 243: 332-9$.

43. Wander SA, Hennessy BT, Slingerland JM. Next-generation mTOR inhibitors in clinical oncology: how pathway complexity informs therapeutic strategy.J Clin Invest 2011; 121 : 1231-41.

44. Das F, Ghosh-Choudhury N, Dey N, et al. Unrestrained Mammalian target of rapamycin complexes 1 and 2 increase expression of phosphatase and tensin homolog deleted on chromosome 10 to regulate phosphorylation of akt kinase. J Biol Chem 2012 ; 287 : 3808-22.

45. Zhang BB, Zhou G, Li C. AMPK: an emerging drug target for diabetes and the metabolic syndrome. Cell Metab 2009; 9 : 407-16.

46. Reiter AK, Bolster DR, Crozier SJ, et al. Repression of protein synthesis and mTOR signaling in rat liver mediated by the AMPK activator aminoimidazole carboxamide ribonucleoside. Am J Physiol Endocrinol Metab 2005; 288 : ع980-8.

47. Natali A, Ferrannini $\varepsilon$. Effects of metformin and thiazolidinediones on suppression of hepatic glucose production and stimulation of glucose uptake in type 2 diabetes: a systematic review. Diabetologia $2006 ; 49$ : 434-4l.

48. Galas $S$, Château MT, Pomiès $P$, et al. Aperçu de la diversité des modèles animaux dédiés à l'étude du vieillissement. Med Sci (Paris) 2012 ; 28 : 297 304

49. Terret C, Solari F. L'homéostasie métabolique au cœur du vieillissement. Med Sci (Paris) $2012 ; 28: 311-5$.

50. Marchal J, Perret $M$, Aujard F. Pas de longévité accrue chez les macaques Rhésus soumis à une restriction calorique. Med Sci (Paris) $2012 ; 28: 1074$

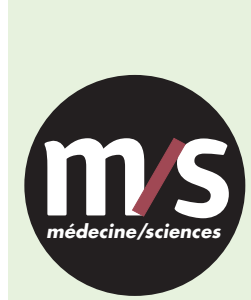

$>$ Grâce à $\mathrm{m} / \mathrm{s}$, vivez en direct

Tarifs d'abonnement $\mathrm{m} / \mathrm{s}-2013$

les progrès des sciences biologiques

et médicales

Abonnez-vous

à médecine/sciences

Bulletin d'abonnement page 1138 dans ce numéro de $\mathrm{m} / \mathrm{s}$

TIRÉS À PART

F. Aujard 